\title{
Improving insulin sensitivity, liver steatosis and fibrosis in type 2 diabetes by a food-based digital education-assisted lifestyle intervention program: a feasibility study
}

\author{
Oana P. Zaharia ${ }^{1,2} \cdot$ Yuliya Kupriyanova $^{1,2} \cdot$ Yanislava Karusheva $^{1,2} \cdot$ Daniel F. Markgraf ${ }^{1,2} \cdot$ Konstantinos Kantartzis $^{2,3}$. \\ Andreas L. Birkenfeld ${ }^{2,3} \cdot$ Michael Trenell $^{4,5} \cdot$ Aarti Sahasranaman $^{5} \cdot$ Chris Cheyette $^{6} \cdot$ Theresa Kössler $^{1,2,7}$. \\ Kálmán Bódis $^{1,2,7} \cdot$ Volker Burkart $^{1,2}$ • Jong-Hee Hwang ${ }^{1,2} \cdot$ Michael Roden $^{1,2,7}$. Julia Szendroedi,2,7 (D) \\ Dominik H. Pesta ${ }^{1,2}$
}

Received: 26 August 2020 / Accepted: 16 February 2021 / Published online: 11 April 2021

(c) The Author(s) 2021

\begin{abstract}
Purpose Recent trials demonstrated remission of type 2 diabetes and non-alcoholic fatty liver disease (NAFLD) following formula diet-induced weight loss. To improve the outreach for populations in need, many mobile health apps targeting weight loss have been developed with limited scientific evaluation of these apps. The present feasibility study investigated the effects of a novel approach incorporating a regular 'whole food-based' low-calorie diet combined with app-based digital education and behavioral change program on glucose metabolism and disease management.

Methods Twenty-four individuals with type 2 diabetes followed this approach supported by weekly coaching calls for 12 weeks. Phenotyping included bioimpedance analysis, mixed-meal tolerance test, magnetic resonance spectroscopy and transient elastography for assessing liver fat content and liver stiffness.

Results Over 12 weeks, participants reduced their body weight by $9 \%$ ( $97 \pm 13$ to $88 \pm 12 \mathrm{~kg}$ ), body mass index (BMI; $33 \pm 5$ to $\left.29 \pm 4 \mathrm{~kg} / \mathrm{m}^{2}\right)$, total fat mass $(31 \pm 10$ to $27 \pm 10 \%$ ) (all $p<0.01)$ and liver fat by $50 \%$ alongside with decreased liver stiffness. Target HbA1c $(<6.5 \%)$ was achieved by $38 \%$ and resolution of NAFLD (liver fat content $<5.6 \%$ ) was observed in $30 \%$ of the participants.

Conclusion This novel approach combining digital education with a low-calorie diet results in effective improvements of body weight, glycemic control and NAFLD and could complement existing care for patients with type 2 diabetes.

Trial registration NCT04509245
\end{abstract}

Keywords Digital education - Type 2 diabetes $\cdot$ Diabetes management $\cdot$ Insulin sensitivity $\cdot$ Non-alcoholic fatty liver disease

Julia Szendroedi

julia.szendroedi@ddz.de

1 Institute for Clinical Diabetology, German Diabetes Center, Leibniz Center for Diabetes Research at Heinrich Heine University, Düsseldorf, Germany

2 German Center for Diabetes Research (DZD), München-Neuherberg, Germany

3 Department of Internal Medicine, Division of Diabetology, Endocrinology, and Nephrology and Institute of Diabetes
Research and Metabolic Diseases (IDM), University Hospital Tübingen, Tübingen, Germany

4 NIHR Innovation Observatory, Newcastle University, Newcastle Upon Tyne, UK

5 Changing Health, Newcastle Upon Tyne, UK

6 Carbs and Cals Ltd, London, UK

7 Division of Endocrinology and Diabetology, Medical Faculty, Heinrich Heine University, c/o Auf'm Hennekamp 65, 40225 Düsseldorf, Germany 


\section{Introduction}

Increased consumption of energy-dense foods, obesity and non-alcoholic fatty liver disease (NAFLD), characterized by hepatocellular lipid content (HCL) of more than $5.6 \%$, are the major metabolic drivers for development of type 2 diabetes [1-3]. Risk of NAFLD progression is higher in patients with type 2 diabetes, which in turn increases other diabetes-related complications, such as cardiovascular morbidity and mortality [4]. Moderate weight loss can halt the vicious cycle by normalizing hepatic steatosis, insulin resistance and hyperglycemia [5]. This underlines the clinical relevance of intensive dietary management also in NAFLD [6]. Current nutritional treatment guidelines for adults with type 2 diabetes focus on improving glycemic control, body weight management and cardiovascular risk factors. Although these guidelines emphasize individualized nutritional therapy, recommendations generally promote healthy whole foods with high fiber content and low added sugars and refined grains [7,8]. With regard to NAFLD, dietary recommendations mostly follow guidelines for adults with obesity and/or type 2 diabetes with a focus on energy restriction and diet quality $[9,10]$. Along these lines, the Mediterranean diet has been suggested as a successful lifestyle recommendation for NAFLD [11]. Dietary energy restriction by complete food replacement via formula diets is also able to induce diabetes remission and normalization of HCL in a subset of patients $[12,13]$. However, it remains unknown whether similar effects can be achieved with a regular, non-formula based diet in connection with digital health education and coaching. Latest obesity management guidelines advocate for the implementation of emerging technologies and virtual medicine incorporating online education, coaching as well as weight and activity tracking to improve weightloss outcomes [14]. Although numerous applications are available for weight loss and diabetes management and mobile health interventions hold great promise to optimize treatment strategies for several chronic diseases, many studies using such applications (apps) are of insufficient quality $[15,16]$. There is also room for improvement as the functionality of some apps is limited and app development is rarely evidence-based [17]. Currently, it is unclear whether stand-alone use of mobile health apps is sufficient or whether adjuncts, such as phone counselling, should be used concomitantly to improve their efficacy for body weight management. To take account of these novel developments and challenges derived thereof, the aim of this feasibility study was to assess whether a combined approach using a 'real food-based' low-calorie diet in connection with a smartphone app and weekly coaching calls for patients with diabetes results in improved body weight, metabolism and disease management.

\section{Subjects and methods}

\section{Participants}

We included 24 patients with type 2 diabetes from the German Diabetes Study (GDS) with less than 4 years disease duration and not receiving insulin. Participants were recruited between 10/2018 and 07/2019 and were included after giving informed consent to the study protocols [18] (Fig. 1).

\section{Intervention}

Before the start of the intervention, participants documented their dietary intake by filing a food diary for 3 days. During the 12-week intervention, participants were asked to adhere to a real food-based low-calorie diet supported by an app-guided digital education program as well as a low-calorie recipe book [19] and received weekly coaching calls by specifically trained nutritionists. The recipe book provides a variety of individual meals and their caloric content and serves as a guide for lowcalorie meal choices for the participants [19]. Patients were instructed to adhere to a balanced low-calorie diet (average $850 \mathrm{kcal} / \mathrm{day}$ ) consisting mainly of high-protein foods with low glycemic index of their choice. Throughout the study, participants photographically documented every food item they consumed. These images were acquired by smartphone through the app and uploaded to an online portal, where they could be accessed and evaluated by the coaches. Based on these images, portion size as well as food items and caloric intake was estimated by trained

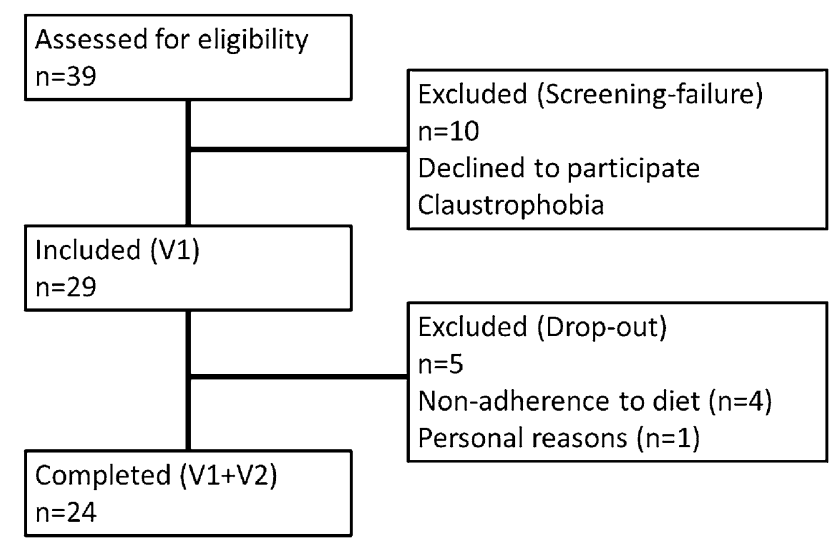

Fig. 1 Recruitment flow diagram 
nutritionists by randomly choosing two days throughout the study. Habitual food intake prior to study inclusion was estimated using 3-day dietary protocols and data were analyzed using the Prodi system (Prodi 6.3.0.1, Nutri-Science $\mathrm{GmbH})$. During the weekly coaching calls, trained coaches provided structured behavior change and motivated the participants to follow a healthy diet, photographically document their daily dietary intake and tracking their body weight, at least on a weekly basis, via the app.

\section{Digital health application}

The Changing Health App (Changing Health, Newcastle, UK) was designed specifically for type 2 diabetes and has been used before in pilot studies in the United Kingdom and the Netherlands. The content of the app also underwent medical and ethical review (ethics boards Heinrich-Heine-University Düsseldorf, reference number 4508). At first visit, patients installed the app and completed an app-based digital education program, consisting of type 2 diabetes education and behavioral change accompanied by weight tracking and dietary monitoring. Patients used the app throughout the study and adherence was monitored based on logins. Participants were advised not to change their physical activity behavior during the intervention. To monitor their activity, daily steps were recorded via the app. Glucose lowering medication was paused three days prior to metabolic tests.

\section{Metabolic testing}

Comprehensive phenotyping before and after the intervention after an overnight fast included a mixed-meal tolerance test (MMTT; Resource Protein, Nestlé Health Science) yielding the oral glucose insulin sensitivity (OGIS) index. The MMTT provides an estimate of endogenous insulin secretion and insulin sensitivity [20]. The ingested drink mimics a conventional meal delivering $463 \mathrm{kcal}$ (macronutrient composition: $13 \mathrm{~g}$ fat, $52 \mathrm{~g}$ carbohydrates $(30 \mathrm{~g}$ sugar) and $35 \mathrm{~g}$ protein). Blood samples for MMTT were taken every $30 \mathrm{~min}$ over $180 \mathrm{~min}$ for measuring glucose, insulin and C-peptide [18]. Fat mass and fat-free mass were assessed by bioimpedance analysis (BioElectrical Impedance Analyzer System, RJL Systems, Detroit, MI, USA). Proton magnetic resonance spectroscopy was performed on a 3-T MR scanner (Achieva X-series, Philips Healthcare) for measurement of HCL expressed as \% to water signal [21]. FibroScan (Roll-stand 530, Echosens, Paris, France) was performed by a trained physician using a minimum of 10 valid measurements (interquartile range $<0.30$ ) for liver stiffness (E) and Controlled Attenuation Parameter (CAP) to assess the degree of steatosis [22].

\section{Ethics}

The study is approved by local institutional review boards (ethics boards Heinrich-Heine-University Düsseldorf, reference number 4508, amendment 20, and University Hospital Tübingen reference number $385 / 2018 \mathrm{BO} 2$ ). This trial was retrospectively registered with ClinicalTrials.gov Identifier: NCT04509245 and was performed according to the Declaration of Helsinki.

\section{Statistics}

Data are presented as mean \pm standard deviation. $P$-values $<0.05$ were considered to indicate statistically significant differences. Pearson correlation coefficient was used to assess associations between metabolic parameters. Paired $\mathrm{t}$-tests were used to assess differences between baseline and post-intervention follow-up in the study population. Normal distribution was assessed by Shapiro-Wilk test. Assuming a baseline HCL of $8.6 \pm 8.2$ (mean $\pm \mathrm{SD}$ ) [23] power calculations estimated reaching a $\beta$ of $90 \%$ by including 24 participants if the true HCL reduction would equal 7 percentage points. Statistical analyses were performed with SAS (version 9.4; SAS Institute, Cary, USA) and GraphPadPrism (version 7.03; GraphPad Software, San Diego, USA).

\section{Results}

Main anthropometric and metabolic parameters are presented in Table 1. In brief, the present study included 14 female and 10 male participants with type 2 diabetes and a mean age of $56 \pm 8$ years. Participants decreased their body weight by $9 \%$ from $97 \pm 14$ to $88 \pm 12 \mathrm{~kg}$, BMI from $33 \pm 5$ to $29 \pm 4 \mathrm{~kg} / \mathrm{m}^{2}$ and fat mass from $31 \pm 10$ to $27 \pm 10 \%$ (all $p<0.01$ ). Caloric intake decreased on average from $1753 \pm 408$ to $868 \pm 108 \mathrm{kcal} /$ day $(p<0.01)$. The approximate macronutrient composition of the diet was roughly $40 \%$ energy from fat, $28 \%$ energy from protein and 32\% energy from carbohydrates. Participants also improved their glycemic control, fasting glucose and OGIS (Fig. 2a-c). Similar results were observed for metabolic changes during MMTT (Fig. 3). In brief, the area under the curve for circulating glucose, insulin and triglycerides during the $180 \mathrm{~min}$ of the MMTT was lower post-intervention compared to study inclusion (all $p<0.05$ ). There was no change in plasma free fatty acid levels during the MMTT.

Diastolic, but not systolic blood pressure decreased alongside with a reduction in resting heart rate (Fig. 2d-f). HCL, alanine aminotransferase activity and liver stiffness 
Table 1 Baseline and follow-up characteristics of patients with type 2 diabetes $(n=24)$

\begin{tabular}{|c|c|c|c|c|}
\hline Parameter & Baseline & Follow-up & Change (\%) & $p$ value \\
\hline Age (years) & $56 \pm 8$ & $56 \pm 8$ & - & - \\
\hline Sex ( $\%$ female $)$ & 58 & 58 & - & - \\
\hline Body weight (kg) & $97.0 \pm 13.9$ & $87.7 \pm 12.1$ & -10 & $<0.01$ \\
\hline BMI $\left(\mathrm{kg} \cdot \mathrm{m}^{-2}\right)$ & $32.6 \pm 4.6$ & $29.4 \pm 3.9$ & -10 & $<0.01$ \\
\hline Fat mass (\%) & $30.9 \pm 9.5$ & $27.4 \pm 10.2$ & -11 & $<0.01$ \\
\hline Fasting C-peptide (ng.ml ${ }^{-1}$ ) & $3.3 \pm 1.7$ & $2.5 \pm 1.0$ & -24 & $<0.05$ \\
\hline Insulin AUC MMTT (a.u.) & $6969 \pm 3899$ & $4868 \pm 2398$ & -30 & $<0.05$ \\
\hline Total cholesterol (mg. $\mathrm{dl}^{-1}$ ) & $185.9 \pm 42.0$ & $174.4 \pm 40.6$ & -6 & 0.09 \\
\hline LDL-cholesterol (mg.dl ${ }^{-1}$ ) & $116.9 \pm 39.6$ & $109.6 \pm 40.4$ & -6 & 0.23 \\
\hline HDL-cholesterol $\left(\mathrm{mg} \cdot \mathrm{dl}^{-1}\right)$ & $48.2 \pm 14.0$ & $50.4 \pm 11.7$ & +5 & 0.09 \\
\hline Fasting triglycerides $\left(\mathrm{mg} \cdot \mathrm{dl}^{-1}\right)$ & $197.0 \pm 127.8$ & $122.7 \pm 60.8$ & -38 & $<0.01$ \\
\hline $\operatorname{AST}\left(\mathrm{U}^{-1} \mathbf{1}^{-1}\right)$ & $25.5 \pm 11.4$ & $23.3 \pm 9.5$ & -9 & 0.21 \\
\hline GGT $\left(\mathrm{U} .1^{-1}\right)$ & $53.3 \pm 72.2$ & $36.5 \pm 47.1$ & -32 & $<0.05$ \\
\hline hsCRP (mg.dl ${ }^{-1}$ ) & $0.4 \pm 0.5$ & $0.6 \pm 1.0$ & +50 & 0.33 \\
\hline TSH $\left(\mu\right.$ IE.ml $\left.^{-1}\right)$ & $1.9 \pm 1.1$ & $1.3 \pm 0.9$ & -32 & $<0.05$ \\
\hline $\mathrm{CAP}\left(\mathrm{dB} \cdot \mathrm{m}^{-1}\right)$ & $326 \pm 64$ & $263 \pm 56$ & -19 & $<0.01$ \\
\hline
\end{tabular}

Data are given as mean \pm standard deviation or percentages, paired samples $t$-test was used for pre-post comparison

$A S T$ aspartate aminotransferase, $A U C$ area under the curve, $B M I$ body mass index, $C A P$ controlled attenuation parameter, $G G T$ gamma glutamyl-transferase, $H D L$ high-density lipoproteins, $h S C R P$ high-sensitivity C-reactive protein, $L D L$ low-density lipoproteins, MMTT mixed-meal tolerance test, TSH thyroid-stimulating hormone

Significant differences as determined by paired samples $t$-test also decreased with the intervention (Fig. $2 \mathrm{~g}-\mathrm{i}$ ). Changes in HCL correlated with alterations in fasting glucose $(r=0.56$, $p=0.006)$, changes in HbA1c $(r=0.59, p=0.003)$ and baseline fasting glucose $(r=0.41, p=0.04)$ but did not correlate with changes in body weight or fat mass. No hypoglycemic episodes or other adverse events were reported during the intervention. The number of steps taken by the participants during the intervention remained constant and equaled on average $\sim 7000$ steps/day.

\section{Discussion}

This feasibility study demonstrates that a combined approach utilizing a 'real food'-based low-calorie diet, supported by digital tools, is viable and yields significant changes in body weight, liver health and glucose metabolism in people with type 2 diabetes. On average, one in three participants normalized their HbAlc and HCL content over a 12 week period. This approach offers an alternative to meal replacements to achieve type 2 diabetes remission. Recent data demonstrated that significant weight loss $(>10 \%)$ is associated with a restoration of pancreatic function and normalization of HbA1c [12]. In these studies, weight loss was achieved using total dietary replacement, targeting $850 \mathrm{kcal} /$ day over a 12 -week period $[12,13]$. Whilst clinically effective, costs and logistics of supplying meal replacements for every meal for a patient over a 12-week period may be prohibitive. Here, we demonstrate the feasibility of a 'real food'-based low-calorie approach, supported by digital tools, in achieving a $10 \%$ weight loss and accompanying metabolic benefits. According to guidelines, people with type 2 diabetes should aim at $>5 \%$ weight loss by intensive lifestyle interventions [24]. With the present approach, nine in ten participants (90\%) achieved or surpassed this goal with one in three (38\%) reaching target HbA1c (criterion $<6.5 \%$ ). Our population was not yet on insulin therapy, which made it safe and feasible to implement a low-calorie diet without changes of medication throughout the study, thus minimizing hypoglycemia risk.

Smartphone applications to supported health interventions are promising tools for individuals with diabetes, likely improving diabetes care and disease self-management [25]. We extend the use of this novel approach in patients with diabetes-related NAFLD.

Excessive caloric intake rich in saturated fats, refined carbohydrates and/or sugar-sweetened beverages, has been implicated in the development of NAFLD [26-28] to occur via increased HCL $[3,26]$. But diets containing saturated fat, not only promote hepatic triglyceride accumulation, but also favor gluconeogenesis, resulting from allosteric activation of pyruvate carboxylase flux and increased glycerol flux, and induce insulin resistance by lipid-mediated inhibition of insulin signaling [2]. Consequently, lower caloric intake 

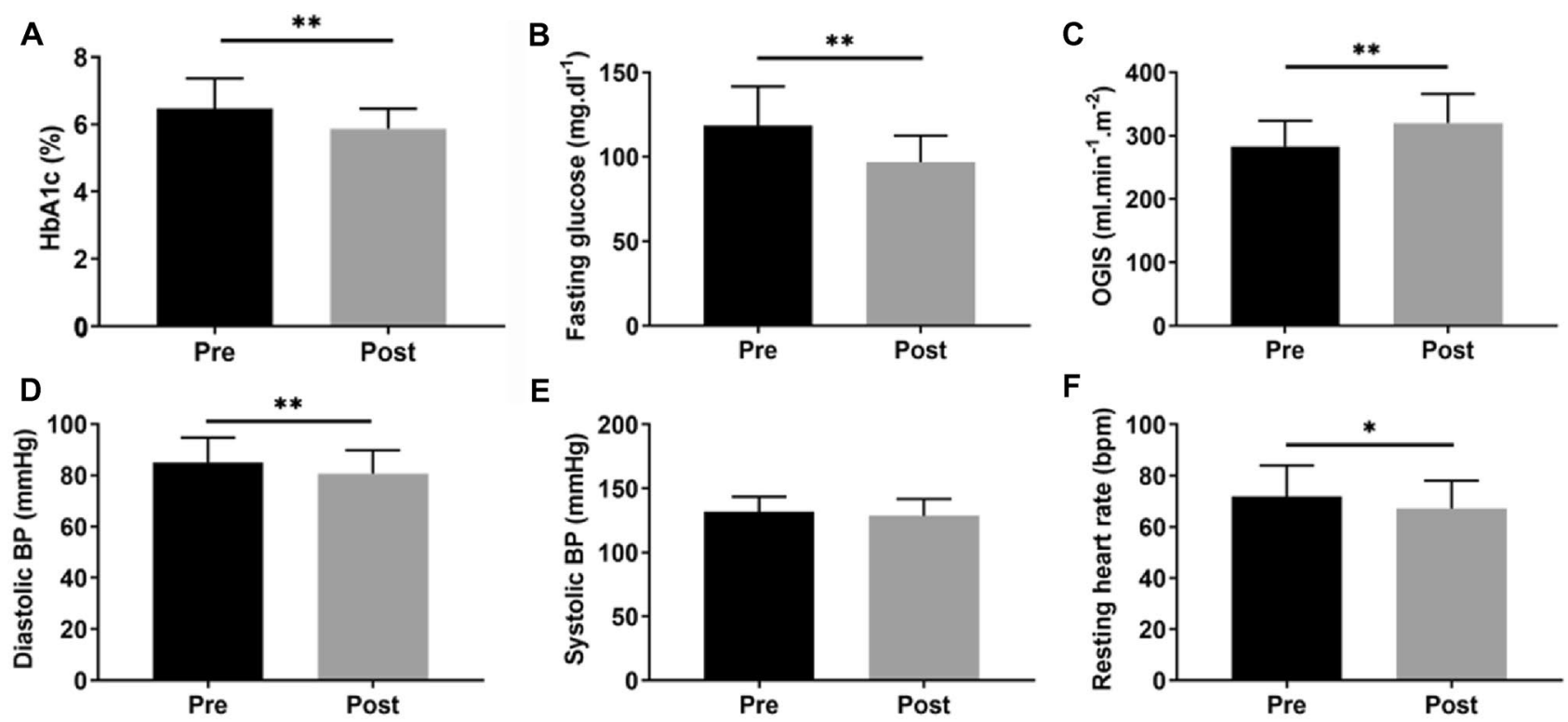

$\mathrm{E}$

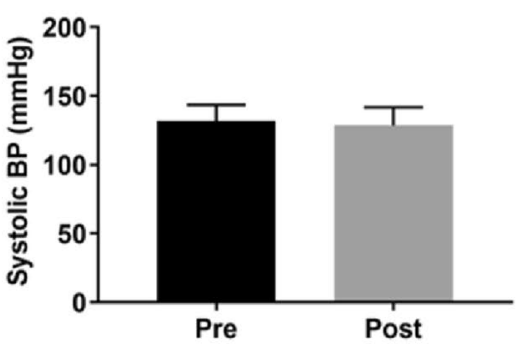

$\mathbf{F}$
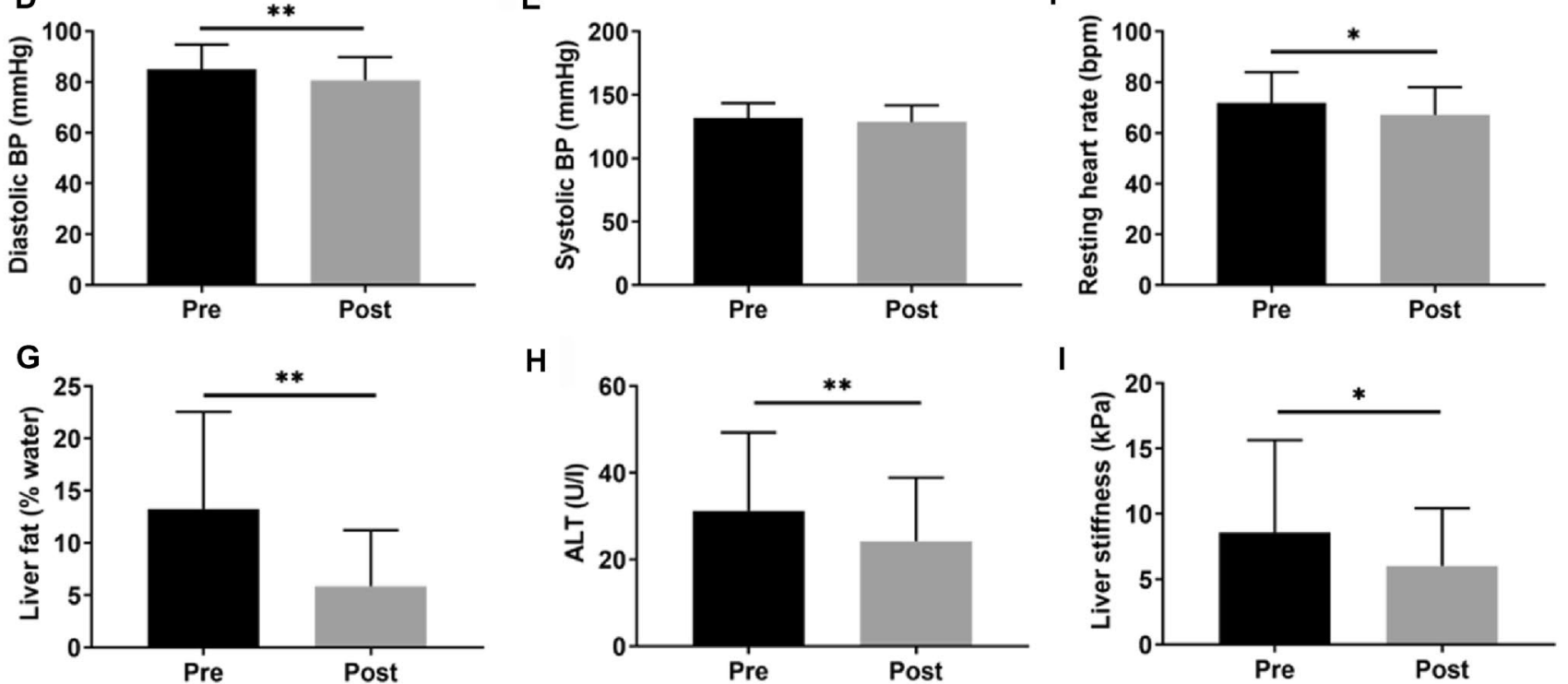

Fig. 2 Metabolic parameters before and after the intervention. Glycemic control (a, b) and insulin sensitivity $(\mathbf{c})$, cardiovascular parameters: blood pressure (d, e) and resting heart rate (f) and liver parameters: HCL (g), ALT (h) and stiffness (i). Black bars represent baseline data while post-intervention data are shown as grey bars. ${ }^{*} p<0.05$;

$*^{*} p<0.01$. Significant differences as determined by paired samples $t$-test for pre-post comparison. $A L T$ alanine aminotransferase, $B P$ blood pressure, HbAlc glycated hemoglobin, OGIS oral glucose insulin sensitivity index

and improved nutrient quality shall reduce HCL, as already described for Mediterranean diets [11]. The Mediterranean diet is characterized by reduced carbohydrate-particularly sugars and refined carbohydrates - and saturated fat content and increased monounsaturated and omega-3 fatty acid content. Body weight loss of $10 \%$ is necessary to improve or reverse NAFLD [1]. Negative energy balance was induced via dietary improvements monitored by coaches based on logged dietary intake data from participants. This likely contributed to the relevant loss of HCL, associating improved glucose metabolism, insulin sensitivity and liver function [13]. Of note, loss of body weight did not associate with HCL reductions. Within this time frame, other factors, such as decreasing glucotoxicity and hyperinsulinemia, may affect reductions in HCL in patients with type 2 diabetes. Decreased fasting triglyceride levels likely aided improved insulin sensitivity. Similarly, NAFLD emerged as a major risk factor for end-stage liver disease, as mortality increases exponentially with fibrosis progression [29]. In the absence of an established pharmacological therapy, lifestyle modification including dietary adjustments is key for preventing excess liver-related mortality [6]. After 12 weeks, about 1/3 of the participants normalized their HCL (criterion $<5.6 \%$ ) [1]. Our approach shows promising results in reducing both HCL and liver fibrosis in patients with diabetes-related NAFLD. This is particularly relevant for people with newly diagnosed type 2 diabetes, who exhibit a rise in HCL during the early course of disease, likely resulting from enlarging adipose tissue volume and insulin resistance in the face of impaired hepatic mitochondrial adaptation [30]. Reduced energy availability possibly facilitated a drop in thyroidstimulating hormone [31]. Patients also improved diastolic $\mathrm{BP}$ and lowered HR, rendering subsequent cardio-metabolic protection to these patients at elevated risk of cardiovascular morbidity and mortality [32]. Participants did not change their physical activity behavior during the intervention as evidenced by a constant step count of about 7000 steps/day. This is comparable with estimates from other 

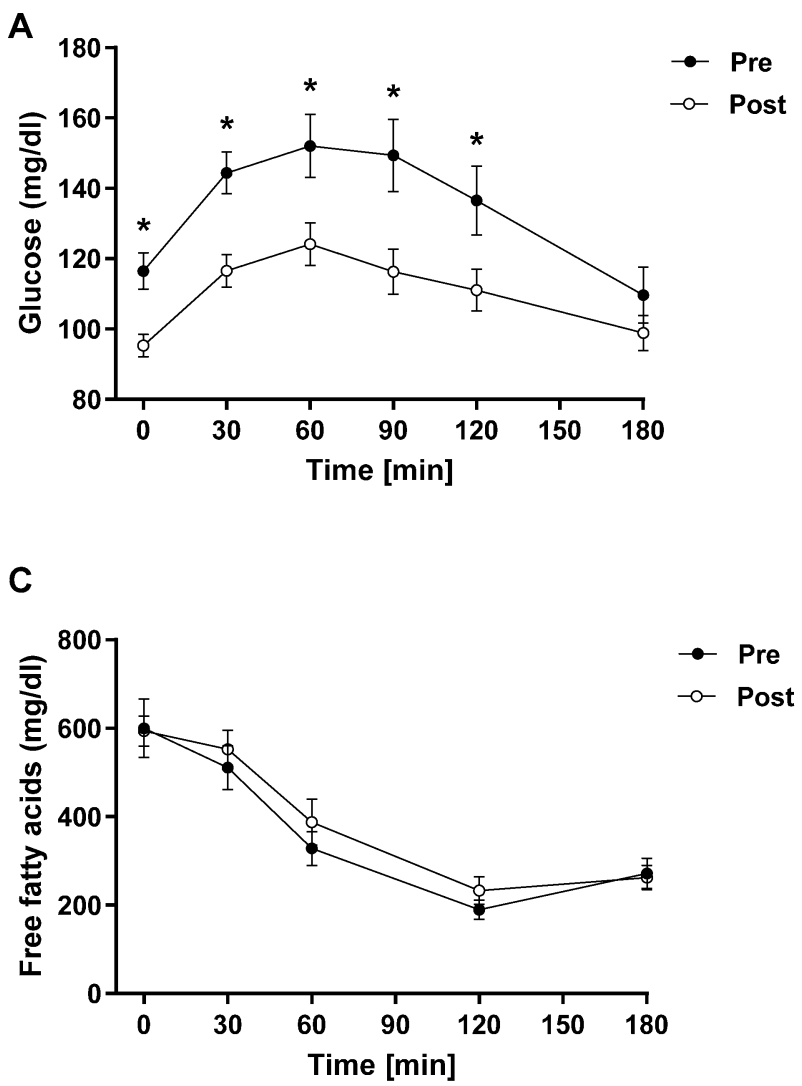

Fig. 3 Metabolic response to mixed-meal tolerance test before and after intervention. Metabolic parameters during the mixed-meal test before and after the intervention: glycemia (a), insulin secretion (b), free fatty acids (c) and triglycerides (d). Black dots and respective

studies evaluating similar patient cohorts, such as adults with impaired glucose tolerance or overt diabetes [33, 34].

The strength of our study results from the comprehensive metabolic characterization using gold-standard methodology [18]. Telephone coaching is potentially suitable for patients in remote areas not served by specialized centers to improve diabetes self-management. However, the longterm costs and sustainability of this intervention remain to be investigated. This study did not include a control group with standard care as it represents a feasibility study testing for the first time the viability and efficacy of this app-based approach in patients with type 2 diabetes. Furthermore, it is not possible to differentiate between the effects of the individual components of the approach, and the results must be interpreted in the context of the multifaceted intervention (low-calorie diet in combination with app-based educational support and photographic dietary diary with weekly coaching). As with any dietary intervention study, a possible rebound-effect after study completion cannot be excluded. Participants were instructed to continue an isocaloric diet in order maintain their post-intervention body weight, yet the
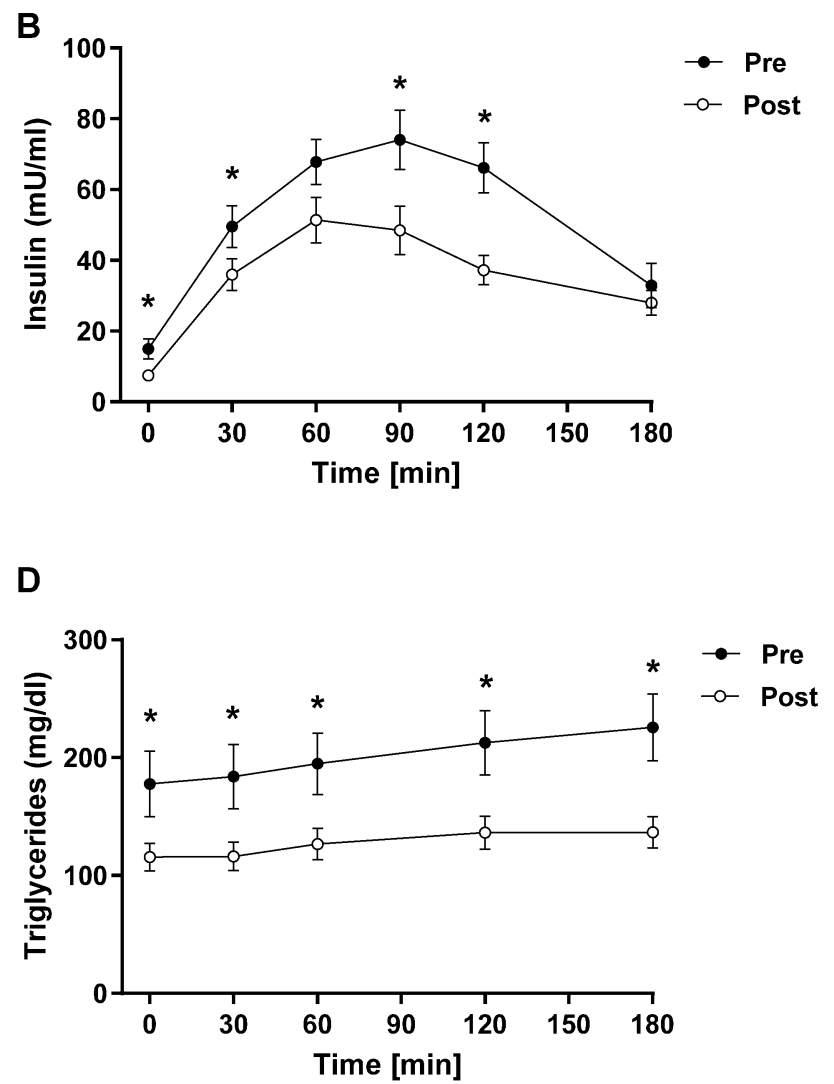

error bars represent baseline data while post-intervention data are shown as white dots and respective error bars. $* p<0.05$. Significant differences as determined by paired samples $t$-test for pre-post comparison

lack of long-term monitoring after the intervention constitutes a limitation of the study.

Taken together, this study shows that an approach combining a real food-based low-calorie diet supported by an app-guided digital education program for patients with diabetes results in clinically relevant reductions in body weight, fat mass, glycemic control and indicators of NAFLD. This approach represents a promising tool for managing patients with type 2 diabetes.

Acknowledgements The authors thank the staff involved in the conduct of the study for their excellent technical help and support. Special thanks to Lisa Nießen and Josefine Hinz for coaching the patients.

Author contributions OPZ wrote the first draft of the manuscript and researched data. YK, TK, YK, KB, KK, DFM, researched data. AS and CC provided app-related support. JS, J-HH, VB, MT, ALB, KK, MR, DHP researched data, contributed to the discussion and reviewed/edited the manuscript. All authors critically reviewed the manuscript. DHP, JS and MR are the guarantors of this work and, as such, had full access to all the data in the study and take responsibility for the integrity of the data and the accuracy of the data analysis. All authors read and approved the final manuscript. 
Funding Open Access funding enabled and organized by Projekt DEAL. This project was supplementary financed by the European Institute of Innovation \& Technology (EIT). The German Diabetes Study was initiated and financed by the German Diabetes Center (DDZ), which is funded by the German Federal Ministry of Health and the Ministry of Culture and Science of the state North Rhine-Westphalia and from the German Federal Ministry of Education and Research (BMBF) to the German Center for Diabetes Research (DZD) and the Schmutzler Stiftung, Germany.

Data availability The data sets generated and/or analyzed during the current study are not publicly available, since they are subject to national data protection laws and restrictions imposed by the ethics committee to ensure data privacy of the study participants. However, they can be applied for through an individual project agreement with the principal investigator of the German Diabetes Study.

\section{Compliance with ethical standards}

Conflict of interest Michael Roden serves as investigator of studies supported by Boehringer-Ingelheim Pharma, Nutriticia / Danone and Sanofi and has served as advisor/consultant for Bristol-Myers Squibb, Eli Lilly, Gilead, Intercept Pharma, Novo Nordisk, Novartis, Poxel, Prosciento, Sanofi, Servier and TARGET NASH. Michael Trenell is co-founder of Changing Health Ltd, a spin-out company from Newcastle University and Newcastle upon Tyne NHS Hospitals Trust. Chris Cheyette authored the recipe book 'Carbs \& Cals' and is managing director at Chello Publishing Limited.

Ethics approval The study was approved by local institutional review boards (ethics boards Heinrich-Heine-University Düsseldorf, reference number 4508, amendment 20, and University Hospital Tübingen reference number $385 / 2018 \mathrm{BO} 2$ ), registered with clinical trials.gov NCT04509245 and was performed according to the Declaration of Helsinki. All persons gave their informed consent prior to their inclusion in the study.

Open Access This article is licensed under a Creative Commons Attribution 4.0 International License, which permits use, sharing, adaptation, distribution and reproduction in any medium or format, as long as you give appropriate credit to the original author(s) and the source, provide a link to the Creative Commons licence, and indicate if changes were made. The images or other third party material in this article are included in the article's Creative Commons licence, unless indicated otherwise in a credit line to the material. If material is not included in the article's Creative Commons licence and your intended use is not permitted by statutory regulation or exceeds the permitted use, you will need to obtain permission directly from the copyright holder. To view a copy of this licence, visit http://creativecommons.org/licenses/by/4.0/.

\section{References}

1. European Association for the Study of the L (2016) EASL-EASDEASO clinical practice guidelines for the management of nonalcoholic fatty liver disease. Obes Facts 9(2):65-90. https://doi. org/10.1159/000443344

2. Roden M, Shulman GI (2019) The integrative biology of type 2 diabetes. Nature 576(7785):51-60. https://doi.org/10.1038/ s41586-019-1797-8

3. Hernandez EA, Kahl S, Seelig A, Begovatz P, Irmler M, Kupriyanova Y, Nowotny B, Nowotny P, Herder C, Barosa C, Carvalho
F, Rozman J, Neschen S, Jones JG, Beckers J, de Angelis MH, Roden M (2017) Acute dietary fat intake initiates alterations in energy metabolism and insulin resistance. J Clin Investig 127(2):695-708. https://doi.org/10.1172/jci89444

4. Zaharia OP, Strassburger K, Strom A, Bonhof GJ, Karusheva Y, Antoniou S, Bodis K, Markgraf DF, Burkart V, Mussig K, Hwang JH, Asplund O, Groop L, Ahlqvist E, Seissler J, Nawroth P, Kopf S, Schmid SM, Stumvoll M, Pfeiffer AFH, Kabisch S, Tselmin S, Haring HU, Ziegler D, Kuss O, Szendroedi J, Roden M, German Diabetes Study G (2019) Risk of diabetes-associated diseases in subgroups of patients with recent-onset diabetes: a 5-year followup study. Lancet Diabetes Endocrinol 7 (9):684-694. doi:https:// doi.org/10.1016/S2213-8587(19)30187-1

5. Petersen KF, Dufour S, Befroy D, Lehrke M, Hendler RE, Shulman GI (2005) Reversal of nonalcoholic hepatic steatosis, hepatic insulin resistance, and hyperglycemia by moderate weight reduction in patients with type 2 diabetes. Diabetes 54(3):603-608

6. El-Agroudy NN, Kurzbach A, Rodionov RN, O'Sullivan J, Roden M, Birkenfeld AL, Pesta DH (2019) Are lifestyle therapies effective for NAFLD treatment? Trends Endocrinol Metab 30(10):701709. https://doi.org/10.1016/j.tem.2019.07.013

7. Evert AB, Dennison M, Gardner CD, Garvey WT, Lau KHK, MacLeod J, Mitri J, Pereira RF, Rawlings K, Robinson S, Saslow L, Uelmen S, Urbanski PB, Yancy WS Jr (2019) Nutrition therapy for adults with diabetes or prediabetes: a consensus report. Diabetes Care 42(5):731-754. https://doi.org/10.2337/dci19-0014

8. Obesity Management for the Treatment of Type 2 Diabetes: Standards of Medical Care in Diabetes-2021 (2021). Diabetes Care 44 (Suppl 1):S100-s110. doi: https://doi.org/10.2337/dc21-S008

9. George ES, Forsyth A, Itsiopoulos C, Nicoll AJ, Ryan M, Sood S, Roberts Stuart K, Tierney AC (2018) Practical dietary recommendations for the prevention and management of nonalcoholic fatty liver disease in adults. Adv Nutr 9(1):30-40. https://doi.org/ 10.1093/advances/nmx007\%JAdvancesinNutrition

10. Miller EF (2020) Nutrition management strategies for nonalcoholic fatty liver disease: treatment and prevention. Clin Liv Dis 15(4):144-148. https://doi.org/10.1002/cld.918

11. Romero-Gómez M, Zelber-Sagi S, Trenell M (2017) Treatment of NAFLD with diet, physical activity and exercise. J Hepatol 67(4):829-846. https://doi.org/10.1016/j.jhep.2017.05.016

12. Lean ME, Leslie WS, Barnes AC, Brosnahan N, Thom G, McCombie L, Peters C, Zhyzhneuskaya S, Al-Mrabeh A, Hollingsworth KG, Rodrigues AM, Rehackova L, Adamson AJ, Sniehotta FF, Mathers JC, Ross HM, McIlvenna Y, Stefanetti R, Trenell M, Welsh P, Kean S, Ford I, McConnachie A, Sattar N, Taylor R (2018) Primary care-led weight management for remission of type 2 diabetes (DiRECT): an open-label, clusterrandomised trial. Lancet 391(10120):541-551. https://doi.org/10. 1016/s0140-6736(17)33102-1

13. Taylor R, Al-Mrabeh A, Zhyzhneuskaya S, Peters C, Barnes AC, Aribisala BS, Hollingsworth KG, Mathers JC, Sattar N, Lean MEJ (2018) Remission of human type 2 diabetes requires decrease in liver and pancreas fat content but is dependent upon capacity for beta cell recovery. Cell Metab 28 (4):547-556 e543. doi:https:// doi.org/10.1016/j.cmet.2018.07.003

14. Wharton S, Lau DCW, Vallis M, Sharma AM, Biertho L, Campbell-Scherer D, Adamo K, Alberga A, Bell R, Boulé N, Boyling E, Brown J, Calam B, Clarke C, Crowshoe L, Divalentino D, Forhan M, Freedhoff Y, Gagner M, Glazer S, Grand C, Green M, Hahn M, Hawa R, Henderson R, Hong D, Hung P, Janssen I, Jacklin K, Johnson-Stoklossa C, Kemp A, Kirk S, Kuk J, Langlois MF, Lear S, McInnes A, Macklin D, Naji L, Manjoo P, Morin MP, Nerenberg K, Patton I, Pedersen S, Pereira L, Piccinini-Vallis H, Poddar M, Poirier P, Prud'homme D, Salas XR, RuedaClausen C, Russell-Mayhew S, Shiau J, Sherifali D, Sievenpiper 
J, Sockalingam S, Taylor V, Toth E, Twells L, Tytus R, Walji S, Walker L, Wicklum S (2020) Obesity in adults: a clinical practice guideline. CMAJ 192 (31):E875-e891. doi:https://doi.org/10. 1503/cmaj.191707

15. Dounavi K, Tsoumani O (2019) Mobile health applications in weight management: a systematic literature review. Am J Prev Med 56(6):894-903. https://doi.org/10.1016/j.amepre.2018.12. 005

16. Ghelani DP, Moran LJ, Johnson C, Mousa A, Naderpoor N (2020) Mobile apps for weight management: a review of the latest evidence to inform practice. Front Endocrinol (Lausanne) 11:412412. https://doi.org/10.3389/fendo.2020.00412

17. Holzmann SL, Holzapfel C (2019) A scientific overview of smartphone applications and electronic devices for weight management in adults. J Pers Med 9(2):31. https://doi.org/10.3390/jpm9020031

18. Szendroedi J, Saxena A, Weber KS, Strassburger K, Herder C, Burkart V, Nowotny B, Icks A, Kuss O, Ziegler D, Al-Hasani H, Mussig K, Roden M, Group GDS (2016) Cohort profile: the German diabetes study (GDS). Cardiovasc Diabetol 15:59. doi:https:// doi.org/10.1186/s12933-016-0374-9

19. Cheyette C, Balolia Y, Francis V, Callaghan S, Turner F (2017) Carbs $\&$ cals very low calorie recipes $\&$ meal plans: lose weight, improve blood sugar levels and reverse type 2 diabetes. chello publishing

20. Besser REJ, Shields BM, Casas R, Hattersley AT, Ludvigsson J (2013) Lessons from the mixed-meal tolerance test: use of 90-min and fasting C-peptide in pediatric diabetes. Diabetes Care 36(2):195-201. https://doi.org/10.2337/dc12-0836

21. Laufs A, Livingstone R, Nowotny B, Nowotny P, Wickrath F, Giani G, Bunke J, Roden M, Hwang JH (2014) Quantitative liver 31P magnetic resonance spectroscopy at $3 \mathrm{~T}$ on a clinical scanner. Magn Reson Med 71(5):1670-1675. https://doi.org/10.1002/mrm. 24835

22. Cassinotto C, Boursier J, de Lédinghen V, Lebigot J, Lapuyade B, Cales P, Hiriart J-B, Michalak S, Bail BL, Cartier V, Mouries A, Oberti F, Fouchard-Hubert I, Vergniol J, Aubé C (2016) Liver stiffness in nonalcoholic fatty liver disease: a comparison of supersonic shear imaging, FibroScan, and ARFI with liver biopsy. Hepatology 63(6):1817-1827. https://doi.org/10.1002/hep.28394

23. Zaharia OP, Strassburger K, Knebel B, Kupriyanova Y, Karusheva Y, Wolkersdorfer M, Bódis K, Markgraf DF, Burkart V, Hwang J-H, Kotzka J, Al-Hasani H, Szendroedi J, Roden M (2020) Role of patatin-like phospholipase domain-containing 3 gene for hepatic lipid content and insulin resistance in diabetes. Diabetes Care 43(9):2161-2168. https://doi.org/10.2337/dc20-0329

24. Obesity Management for the Treatment of Type 2 diabetes: standards of medical care in diabetes-2019 (2019). Diabetes care 42 (Suppl 1):S81-s89. doi:https://doi.org/10.2337/dc19-S008

25. Chavez S, Fedele D, Guo Y, Bernier A, Smith M, Warnick J, Modave F (2017) Mobile apps for the management of diabetes. Diabetes Care 40(10):e145-e146. https://doi.org/10. 2337/dc17-0853

26. Pafili K, Roden M (2020) Nonalcoholic fatty liver disease (NAFLD) from pathogenesis to treatment concepts in humans. Mol Metab:101122. doi:https://doi.org/10.1016/j.molmet.2020. 101122

27. Koopman KE, Caan MW, Nederveen AJ, Pels A, Ackermans MT, Fliers E, la Fleur SE, Serlie MJ (2014) Hypercaloric diets with increased meal frequency, but not meal size, increase intrahepatic triglycerides: a randomized controlled trial. Hepatology 60(2):545-553. https://doi.org/10.1002/hep.27149

28. Cotter TG, Rinella M (2020) Nonalcoholic fatty liver disease 2020: the state of the disease. Gastroenterology 158(7):18511864. https://doi.org/10.1053/j.gastro.2020.01.052

29. Dulai PS, Singh S, Patel J, Soni M, Prokop LJ, Younossi Z, Sebastiani G, Ekstedt M, Hagstrom H, Nasr P, Stal P, Wong VW, Kechagias S, Hultcrantz R, Loomba R (2017) Increased risk of mortality by fibrosis stage in nonalcoholic fatty liver disease: Systematic review and meta-analysis. Hepatology 65(5):1557-1565. https:// doi.org/10.1002/hep.29085

30. Kupriyanova Y, Zaharia OP, Bobrov P, Karusheva Y, Burkart V, Szendroedi J, Hwang JH, Roden M (2020) Early changes in hepatic energy metabolism and lipid content in recent-onset type 1 and 2 diabetes mellitus. J Hepatol. https://doi.org/10.1016/j.jhep. 2020.11.030

31. Aeberli I, Jung A, Murer SB, Wildhaber J, Wildhaber-Brooks J, Knopfli BH, Zimmermann MB (2010) During rapid weight loss in obese children, reductions in TSH predict improvements in insulin sensitivity independent of changes in body weight or fat. J Clin Endocrinol Metab 95(12):5412-5418. https://doi.org/10.1210/jc. 2010-1169

32. Böhm M, Schumacher H, Teo KK, Lonn EM, Mahfoud F, Ukena C, Mann JFE, Mancia G, Redon J, Schmieder RE, Sliwa K, Marx N, Weber MA, Williams B, Yusuf S (2020) Resting heart rate and cardiovascular outcomes in diabetic and non-diabetic individuals at high cardiovascular risk analysis from the ONTARGET/ TRANSCEND trials. Eur Heart J 41(2):231-238. https://doi.org/ 10.1093/eurheartj/ehy808

33. Tudor-Locke C, Bell RC, Myers AM, Harris SB, Ecclestone NA, Lauzon N, Rodger NW (2004) Controlled outcome evaluation of the First Step Program: a daily physical activity intervention for individuals with type II diabetes. Int J Obes Relat Metab Disord 28(1):113-119. https://doi.org/10.1038/sj.ijo.0802485

34. Huffman KM, Sun J-L, Thomas L, Bales CW, Califf RM, Yates T, Davies MJ, Holman RR, McMurray JJV, Bethel MA, Tuomilehto J, Haffner SM, Kraus WE (2014) Impact of baseline physical activity and diet behavior on metabolic syndrome in a pharmaceutical trial: results from NAVIGATOR. Metabolism: clinical and experimental 63 (4):554-561. doi:https://doi.org/10.1016/j. metabol.2014.01.002 